\title{
Incentive compatibility and pricing under moral hazard
}

\author{
Belén Jerez
}

Department of Economics, Universidad Carlos III, Getafe, Madrid 28903, Spain

We show how to recover equilibrium prices supporting incentive-efficient allocations in a classic insurance economy with moral hazard. Our key modeling choice is to impose the incentivecompatibility constraints on insurance firms, and not on consumers as in Prescott and Townsend [Pareto optima and competitive equilibria with adverse selection and moral hazard, Econometrica 52 (1984) 21-45]. We show that equilibrium prices of insurance contracts are equal to the sum of the shadow costs arising from the resource and incentive-compatibility constraints in the planner's problem. The equilibrium allocations are the same as when the incentive-compatibility constraints are imposed on consumers. As in Prescott and Townsend, the two welfare theorems hold.

\section{Introduction}

In their pathbreaking contribution, Prescott and Townsend (1984a, 1984b) show how to extend the Arrow-Debreu model to a large class of economies with asymmetric information. In these economies, asymmetric information is realized ex post, that is after agents have traded. This class includes economies with moral hazard, where agents choose their

This work is part of my PhD dissertation at UCLA, and circulated in an earlier draft with the title "General Equilibrium with Asymmetric Information: A Dual Approach.”

E-mail address: mjerez@eco.uc3m.es. 
effort after they have traded. ${ }^{1}$ In particular, Prescott and Townsend define allocations in the space of lotteries over bundles of state-contingent commodities. They then derive the welfare theorems and show that a competitive equilibrium exists. The key modeling choice of Prescott and Townsend is to impose the incentive-compatibility constraints arising from asymmetric information on consumers, and not on firms. A typical example is an insurance economy with moral hazard where consumers are subject to idiosyncratic risk. As in the full-information benchmark, firms supply actuarially fair insurance plans and any actuarially fair insurance plan is budget feasible. Consumers choose from the actuarially fair insurance plans under the incentive-compatibility constraints. As a result the second best is attained.

The motivation for our paper is a potential conceptual problem with imposing the incentive-compatibility constraints on consumers: it is unclear how these incentivecompatibility constraints are enforced in the decentralized economy. The natural interpretation is to view the incentive-compatibility constraints as restrictions on the set of contracts that firms can offer to consumers, rather than as consumers self-imposing these constraints. $^{2}$ Our paper therefore takes a more natural approach and imposes the incentivecompatibility constraints on firms. As in the standard general equilibrium model, all the relevant information is then conveyed through prices. In particular, equilibrium prices reflect the shadow costs arising from the resource and incentive-compatibility constraints. This result is in contrast with the full information benchmark, where prices reflect only the shadow costs arising from the resource constraints. The equilibrium allocations are the same as when the incentive-compatibility constraints are imposed on consumers. As in Prescott and Townsend (1984a, 1984b), the two welfare theorems hold.

We make our point in a classic moral hazard economy. There is a continuum of ex ante identical consumers and a finite number of idiosyncratic endowment states. Each consumer can exert high or low effort at a direct utility cost. High effort reduces the probability of ending up in a poor state. The commodities are insurance contracts, which are signed between a consumer and a firm. An insurance contract specifies a vector of statecontingent net trades and an effort level for the consumer. We assume that net trades are perfectly verifiable and fully enforceable. It therefore suffices to consider exclusive contractual relations in which consumers can buy insurance from at most one firm. Firms have access to a constant-returns-to-scale insurance technology and they face both technological and incentive-compatibility constraints. The incentive-compatibility constraints require that insurance contracts give the consumers an incentive to conform to the effort specifications. As in Prescott and Townsend (1984a), we allow for lotteries to overcome the non-convexities generated by the incentive-compatibility constraints.

We show how to recover equilibrium prices supporting incentive-efficient allocations. Equilibrium prices are equal to the sum of the shadow costs arising from the resource and incentive-compatibility constraints in the planner's problem. For example, actuarially fair

\footnotetext{
${ }^{1}$ However, it does not include economies with adverse selection, where agents learn their types before they trade (ex ante asymmetric information).

2 In the mechanism design literature, the principal offers a contract subject to the incentive-compatibility constraint of the agents. See also the competitive models with asymmetric information in the partial equilibrium (e.g. Rothschild and Stiglitz, 1976 and Wilson, 1977, and Bennardo and Chiappori, 2003).
} 
contracts which specify a high effort generate identical resource costs but different incentive costs. Providing more insurance implies higher incentive costs because it raises the consumers' incentive to shirk. This raises the equilibrium price of an actuarially fair contract. Consumers then do not purchase the full-insurance contract because it is not budget feasible, and firms do not offer it because it is not incentive compatible (with full insurance consumers always shirk). As a result, the competitive equilibrium allocation provides only partial insurance. The amount of insurance is the same as when the incentive-compatibility constraints are imposed on consumers, and so the second-best is attained.

There are also some formal differences between our approach and that of Prescott and Townsend (1984a). With their approach, equilibrium prices are the same as in the fullinformation benchmark, so they are linear on the agents' net trade sets. With our approach, equilibrium prices are not the same as in the full-information benchmark. Instead, they are non-linear on the agents' net trade sets. The reason is that equilibrium prices reflect the shadow costs arising from the incentive-compatibility constraints, and these shadow costs are non-linear and may even be non-convex. This feature of our model is perfectly consistent with standard general equilibrium analysis, because prices remain linear in the general space of lotteries (the commodity space). A second formal difference with respect to Prescott and Townsend is that the infinite dimensional spaces in which allocations and prices lie are not approximated by finite grids. This is as in Kehoe et al. (2002) for a related exchange economy with ex post private information about endowments. Kehoe, Levine and Prescott (Kehoe et al., 2002) derive the welfare and existence theorems by introducing the notion of the stand-in consumer economy, which is a standard finite convex exchange economy. In addition, they show that a lottery equilibrium allocation can be implemented in a sunspot equilibrium. Our paper differs from their paper because we impose the incentivecompatibility constraints on firms, while they impose them on consumers. Moreover, we derive our results using the techniques of linear programming. ${ }^{3}$

Our application of linear programming draws heavily on the work of Makowski and Ostroy (1996), who develop the linear programming methodology for large economies with full information. Specifically, they use a measure-theoretic description of the economy to show that efficient allocations solve a linear programming problem. Then they establish an equivalence between the competitive equilibrium allocations and prices, on the one hand, and the solutions to the primal and dual problems, on the other hand. Gretsky et al. (1999) present a similar analysis for large assignment economies.

The paper is organized as follows. In Section 2 we describe the economy. In Section 3 we present the general equilibrium model. In Section 4 we define a competitive equilibrium and characterize the competitive equilibrium prices and allocations. In addition, we derive the two welfare theorems and establish the existence of a competitive equilibrium. Section 5 concludes. The proofs are deferred to Appendix A.

\footnotetext{
3 This paper complements the work of a companion paper (Jerez, 2003), where we show that incentive-efficient allocations solve a linear program, and use linear programming techniques to characterize the optima.
} 


\section{The economy}

There is a continuum of identical consumers with measure one and a single consumption good. Consumers are subject to idiosyncratic endowment shocks. Shocks are independent across consumers and render no aggregate uncertainty. ${ }^{4}$ Each consumer faces $S$ idiosyncratic states, $s=1, \ldots, S$. The consumer's endowment in state $s$ is denoted by $\omega_{s}$, and satisfies $\omega_{s} \leqslant \omega_{s^{\prime}}$ if $s<s^{\prime}$ (endowments are lower in lower states). The consumer is moreover endowed with one unit of time that she allocates between leisure activities and effort in preventing the realization of a low state. Effort can be either high or low, with the set of effort levels denoted by $E=\left\{e_{L}, e_{H}\right\}$, where $0 \leqslant e_{L}<e_{H}$. We denote the probability of state $s$ with effort $e_{i}$ by $\theta_{i s}$. We assume that the likelihood ratio $\left\{\theta_{H s} / \theta_{L s}\right\}$ increases with the state $s$. So high effort reduces the probability of ending up in a low state. Consumers have von Neumann-Morgenstern preferences as defined by the utility function $u: E \times \Re_{+} \rightarrow \Re$. The utility of consumption $c$ under effort $e_{i}$ is then given by $U_{i}(c)=u\left(e_{i}, c\right)$, where $U_{i}$ is assumed twice continuously differentiable, strictly increasing, and strictly concave with $\lim _{c \rightarrow 0} U_{i}^{\prime}(c)=\infty$ and $\lim _{c \rightarrow \infty} U_{i}^{\prime}(c)=0$. Effort is costly, so $U_{L}(c)-U_{H}(c)>d$ for all $c \in \Re_{+}$and some positive constant $d$.

There is a finite number of insurance firms which are large relative to the non-atomic consumers. Each firm insures a positive mass of agents, thus facing no aggregate risk. All firms have access to an identical constant-returns-to-scale insurance technology. ${ }^{5} \mathrm{We}$ assume that insurance claims are perfectly verifiable and fully enforceable. It therefore suffices to consider exclusive contractual relations in which consumers can buy insurance from at most one firm. ${ }^{6}$

The timing of the model is as follows. At some initial date, the insurance market opens and consumers buy insurance from the firms. After the trading period, consumers choose their effort level. Then, endowment shocks are realized. Finally, insurance contracts are enforced, and consumption takes place. There is no ex post trade. The structure of uncertainty is common knowledge and the realization of the endowment shocks is observable. However, effort is private information.

\section{The general equilibrium model}

In this section, we describe the commodity space, the consumption and production sets, and the consumers' utility over consumption bundles. We then define allocations. We begin with some preliminary notation.

\footnotetext{
${ }^{4}$ We assume that the law of large numbers holds. See Sun (1998).

5 This assumption implies that each firm is redundant in the economy and has no market power.

6 See Bisin and Gottardi (1999) and Bisin and Guatoli (1997) for the analysis of moral hazard economies with non-exclusive contracts.
} 


\subsection{Notation}

Let $Z$ be the consumer's net trade set, and denote its elements by $z=\left(z_{1}, \ldots, z_{S}\right)$ :

$$
Z=\left\{z \in R^{S}: z_{s} \geqslant-\omega_{s}, s=1, \ldots, S\right\} .
$$

Let $C(Z)$ denote the space of continuous real-valued functions on $Z$, endowed with the topology of uniform convergence on compact sets. The topological dual of $C(Z)$ is the space of signed Borel measures on $Z$ which are finite on compact sets and have compact support. ${ }^{7}$ We denote the dual space by $M_{c}(Z)$, and let it be endowed with the weak-star topology. Then, $C(Z)$ is also the dual of $M_{c}(Z)$. The dual pair of spaces $\left(C(Z), M_{c}(Z)\right)$ is endowed with the standard bilinear form:

$$
\langle f, \mu\rangle \equiv \int_{Z} f(z) \mathrm{d} \mu(z), \quad f \in C(Z), \quad \mu \in M_{c}(Z),
$$

where the bracket notation highlights the infinite dimensional nature of the spaces in the pairing. We denote the total variation of a measure $\mu \in M_{c}(Z)$ by $\|\mu\|$.

\subsection{Commodities}

The commodities are insurance contracts, which are signed between a consumer and a firm. An insurance contracts specifies an effort level $e \in E$ and a vector of state-contingent net trades $z \in Z$. Both specifications are allowed to be random and are given as follows. ${ }^{8}$ First, the consumer is assigned a lottery which specifies an effort level. After the consumer chooses her effort and conditional on the effort specification received, a second lottery specifies a vector of state-contingent net trades.

We take as the commodity space the product space

$$
L=M_{c}(Z) \times M_{c}(Z),
$$

endowed with the product topology. We describe an insurance contract by a bundle $x=$ $\left(x_{L}, x_{H}\right) \in L^{+}$such that

$$
\left\|x_{L}\right\|+\left\|x_{H}\right\|=\int_{Z} \mathrm{~d} x_{L}(z)+\int_{Z} \mathrm{~d} x_{H}(z)=1 .
$$

Here, $\left\|x_{i}\right\|$ represents the probability that the insurance contract specifies effort $e_{i}$, and the equality in (3.1) is an adding-up condition. In addition, the probability measure $x_{i} /\left\|x_{i}\right\|$ represents the random net trade assigned conditional on specification $e_{i}$. Note that the uncertainty involved in a contract resolves in two steps. In the first step, consumers may be uncertain about the effort that the contract will specify. This occurs when both $\left\|x_{L}\right\|$ and $\left\|x_{H}\right\|$ are positive. In the second step, consumers find out their effort specifications but,

\footnotetext{
7 See Hewitt (1959).

8 It is well known since the seminal work of Prescott and Townsend (1984a) that lotteries may play a role in the presence of incentive-compatibility constraints. In Jerez (2003) we derive conditions under which random effort specifications and random net trades are optimal in this model. See also Bennardo and Chiappori (2003).
} 
in deciding whether to conform or not to such specifications, they may still be uncertain about the net trade that the contract will specify (and thus about their state-contingent consumption plan). This occurs when $x_{i} /\left\|x_{i}\right\|$ is a non-degenerate probability measure.

Remark. We could also take as the commodity space the space of compactly supported measures over pairs of effort and net trade, $M_{c}(E \times Z)$. An insurance contract would then be defined as a probability measure on $E \times Z$. The two definitions of the commodity space are equivalent. Our choice of the commodity space has the advantage that it directly implies that incentive-efficient allocations solve a linear programming problem (see Jerez, 2003). Our choice of the commodity space is also equivalent to the one of Prescott and Townsend (1984a), who define the commodity space to be the space of measures over triples of effort, consumption and endowment. The difference with respect to Prescott and Townsend (1984a) is that they assume that the underlying consumption set, and thus net trade set $Z$, is a finite set. With this assumption, the commodity space is finite dimensional since it is isomorphic to the Euclidean space. We consider the general case in which the net trade set need not be a finite set. ${ }^{9}$

\subsection{Consumption sets}

The consumption set $X$ is the set of insurance contracts:

$$
X=\left\{\left(x_{L}, x_{H}\right) \in L^{+}:\left\|x_{L}\right\|+\left\|x_{H}\right\|=1\right\} .
$$

The exclusivity assumption implies that consumers can buy at most one contract. Consumers can choose to be uninsured with $z=0$ and exert any effort level $e_{i}$. In this case, $x_{i}=\delta_{0}$ and $x_{j}=0$ for $j \neq i$ (with $\delta_{0}$ denoting the Dirac measure at $z=0$ ).

\subsection{Preferences}

The expected utility of a consumer with effort $e_{i}$ and net trade $z$ is

$$
E U_{i}(z) \equiv \sum_{s=1}^{S} \theta_{i s} U_{i}\left(\omega_{s}+z_{s}\right) .
$$

The expected utility from an insurance contract $x \in X$ is then ${ }^{10}$

$$
\begin{aligned}
\langle E U, x\rangle & =\left\langle E U_{L}, x_{L}\right\rangle+\left\langle E U_{H}, x_{H}\right\rangle \\
& =\int_{Z} E U_{L}(z) \mathrm{d} x_{L}(z)+\int_{Z} E U_{H}(z) \mathrm{d} x_{H}(z) .
\end{aligned}
$$

Since $x$ is a lottery, the consumer's expected utility is linear.

\footnotetext{
9 See also Kehoe et al. (2002) for a related exchange economy with private information over endowments.

10 Here, $E U=\left(E U_{L}, E U_{H}\right) \in C(Z) \times C(Z)$.
} 


\subsection{Production sets}

Each firm supplies a single insurance contract. ${ }^{11}$ A production plan is described by a bundle $y=\left(y_{L}, y_{H}\right) \in L^{+}$. Here, (i) $y /\|y\|$ is the contract supplied by the firm, and (ii) $\|y\|$ is the total mass of contracts supplied. The law of large numbers implies that, when the firm insures a positive mass of consumers, it faces no uncertainty. We assume that the firm assigns lotteries across consumers in order to preserve this lack of uncertainty. Then $\left\|y_{i}\right\|$ represents the ex post mass of consumers who are specified effort $e_{i}$, and $y_{i} /\left\|y_{i}\right\|$ represents the distribution of the state-contingent net trade vectors of these consumers once the outcomes of their individual lotteries are realized.

The expected net trade of a consumer with effort $e_{i}$ and net trade $z$ is

$$
r_{i}(z) \equiv \sum_{s=1}^{S} \theta_{i s} z_{s} .
$$

The net transfer of resources that the firm makes to its customers under production plan $y$ is then

$$
\left\langle r_{L}, y_{L}\right\rangle+\left\langle r_{H}, y_{H}\right\rangle=\int_{Z} r_{L}(z) \mathrm{d} y_{L}(z)+\int_{Z} r_{H}(z) \mathrm{d} y_{H}(z) .
$$

A production plan $y$ is technologically feasible if the net transfer of resources that the firm makes to its customers is non-positive:

$$
\left\langle r_{L}, y_{L}\right\rangle+\left\langle r_{H}, y_{H}\right\rangle \leqslant 0 .
$$

Since the firm cannot observe the effort choice of its customers, the contract it offers must be incentive compatible. Under production plan $y$, the utility of a consumer who is specified effort $e_{i}$ and chooses effort $e_{j}$ is

$$
\left\langle E U_{j}, \frac{y_{i}}{\left\|y_{i}\right\|}\right\rangle=\frac{1}{\left\|y_{i}\right\|} \int_{Z} E U_{j}(z) \mathrm{d} y_{i}(z) .
$$

A production plan $y$ is incentive compatible if it is not in the interest of the consumers to deviate from their effort specifications:

$$
\left\langle E U_{i}, y_{i}\right\rangle \geqslant\left\langle E U_{j}, y_{i}\right\rangle, \quad j \neq i, i, j=L, H .
$$

The production set $Y$ is the set of production plans satisfying the technological and incentive-compatibility constraints:

$$
\begin{aligned}
Y=\{ & \left\{\left(y_{L}, y_{H}\right) \in L^{+}:\left\langle r_{L}, y_{L}\right\rangle+\left\langle r_{H}, y_{H}\right\rangle \leqslant 0,\right. \\
& \left.\left\langle E U_{i}-E U_{j}, y_{i}\right\rangle \geqslant 0, j \neq i, i, j=L, H\right\} .
\end{aligned}
$$

Since all constraints are linear, the production set $Y$ is convex and displays constant returns to scale (i.e., $Y$ is a convex cone). Since $0 \in Y$, the firm can choose to be inactive.

\footnotetext{
11 Since consumers are ex ante identical, we shall restrict our attention to symmetric allocations.
} 


\section{Competitive equilibrium}

In this section, we define a competitive equilibrium. We then use linear programming techniques to characterize the competitive equilibrium prices and allocations. We begin by describing the price space.

\subsection{Prices}

The price space $P$ is set of continuous linear functionals on the commodity space (the dual space):

$$
P \equiv L^{*}=C(Z) \times C(Z),
$$

endowed with the product topology. A price system is then a pair of continuous functions on $Z$, denoted by $p=\left(p_{L}, p_{H}\right)$. For a given $p \in P$, the value of a commodity bundle $x \in L^{+}$is given by the inner product:

$$
\langle p, x\rangle=\left\langle p_{L}, x_{L}\right\rangle+\left\langle p_{H}, x_{H}\right\rangle=\int_{Z} p_{L}(z) \mathrm{d} x_{L}(z)+\int_{Z} p_{H}(z) \mathrm{d} x_{H}(z) .
$$

In particular, the price of a deterministic contract which specifies effort $e_{i}$ and net trade $z$ is $p_{i}(z){ }^{12}$ That is, prices depend both on the effort and the net trade specified by the contract. On the other hand, a lottery specifies different pairs of effort and net trade with positive probability. Equation (4.11) says that the price of a lottery is calculated by adding the values of each individual component using the corresponding probability weights (i.e. integrating $p_{i}(z)$ over $z$ with respect to the measure $x_{i}$ for each $e_{i}$, and summing over $e_{i}$ ). ${ }^{13}$

Unlike in the full-information benchmark, prices are not necessarily linear in the underlying net trades. Take two deterministic contracts, $x_{1}$ and $x_{2}$, which prescribe the same effort level $e_{i}$ and assign net trades $z$ and $t z$ (with $t>0$ and $t \neq 1$ ). Their respective prices are $p_{i}(z)$ and $p_{i}(t z)$. Critically, however, these prices need not satisfy $p_{i}(t z)=t p_{i}(z)$. That is, even though the net trade assigned by $x_{2}$ in each state is $t$ times the net trade assigned by $x_{1}$, the price of $x_{2}$ need not be $t$ times the price of $x_{1}$. The reason is that the continuous function $p_{i}$ need not be a linear function. ${ }^{14}$ While the possibility of non-linear pricing may seem inconsistent with standard general equilibrium theory, the inconsistency is only apparent. Equation (4.11) shows that prices in the general space of lotteries are constructed as expected values given the prices in the underlying space of degenerate lotteries. Therefore, prices are linear in the general space of lotteries (the commodity space). ${ }^{15}$

12 The deterministic contract is given by $x_{i}=\delta_{z}$ and $x_{j}=0$ (with $\delta_{z}$ denoting the Dirac measure at $z$ ), so its price is: $\langle p, x\rangle=\left\langle p_{i}, x_{i}\right\rangle=\left\langle p_{i}, \delta_{z}\right\rangle=p_{i}(z)$.

13 See also Prescott and Townsend (1984a).

14 The set of price systems $p=\left(p_{L}, p_{H}\right)$ where $p_{L}$ and $p_{H}$ are linear functions is only a subset of the price space $P$.

15 Prices are (i) additive: $\left\langle p, x_{1}+x_{2}\right\rangle=\left\langle p, x_{1}\right\rangle+\left\langle p, x_{2}\right\rangle$ for all $x_{1}, x_{2} \in L^{+}$; and (ii) homogeneous: $\langle p, t x\rangle=$ $t\langle p, x\rangle$ for all $x \in L^{+}$and all $t \in \Re_{+}$. 


\subsection{Competitive equilibrium}

Since the production set displays constant returns to scale, we may assume that there is a single firm in the economy. A competitive equilibrium is defined in the standard way.

Definition 4.1. A competitive equilibrium is an allocation $\left(x^{*}, y^{*}\right) \in L^{2}$ and a price system $p^{*} \in L^{*}$ for which

(i) $x^{*}$ maximizes $\langle E U, x\rangle$ over the set $\left\{x \in X: p^{*} \cdot x \leqslant 0\right\}$;

(ii) $y^{*}$ maximizes $\left\langle p^{*}, y\right\rangle$ over the set $Y$; and

(iii) markets clear, or $x^{*}=y^{*}$.

Condition (i) requires that contract $x^{*}$ yields consumers the highest utility among all budget feasible contracts lying in their consumption set. Condition (ii) requires that $y^{*}$ yields the firm the maximal level of profits within the set $Y$. The market clearing condition (iii) requires that the contract demanded by consumers coincides with the contract supplied by the firm, and that the total mass of contracts supplied by the firm is equal to the total mass of consumers.

In order to characterize the competitive equilibrium prices and allocations, we analyze the optimal decisions of the firm and the consumers. Then we relate these optimal decisions through the market clearing condition.

\subsection{Optimal consumption plans}

The consumer chooses $x=\left(x_{L}, x_{H}\right) \in L$ to solve the following linear programming problem:

$\left(D_{c}\right) \sup \left\langle E U_{L}, x_{L}\right\rangle+\left\langle E U_{H}, x_{H}\right\rangle$

s.t.

$$
\begin{aligned}
& \left\langle\mathcal{I}, x_{L}\right\rangle+\left\langle\mathcal{I}, x_{H}\right\rangle=1, \\
& \left\langle p_{L}, x_{L}\right\rangle+\left\langle p_{H}, x_{L}\right\rangle \leqslant 0, \\
& x_{L}, x_{H} \geqslant 0 .
\end{aligned}
$$

Condition (4.12) is the adding-up condition on the lottery $x$ expressed in bilinear form, with $\mathcal{I}: Z \rightarrow\{0,1\}$ denoting the characteristic function on $Z$. The budget constraint (4.13) says that the value the lottery must be non-positive. ${ }^{16}$

Problem $\left(D_{c}\right)$ is dual to another linear programming problem. The primal problem $\left(P_{c}\right)$, which is derived in detail in Appendix A, consists of finding a pair $\left(\alpha^{c}, \lambda\right) \in \mathbf{R}^{2}$ to solve

$\left(P_{c}\right) \inf \alpha^{c}$

\footnotetext{
$\overline{16}$ Since a lottery specifies a random pair of effort and net trade, this constraint is analogous to the full-
} information budget constraint, according to which the value of the consumer's net trade must be non-positive. 
s.t.

$$
\begin{aligned}
& \alpha^{c} \geqslant E U_{L}(z)-\lambda p_{L}(z) \quad \forall z \in Z, \\
& \alpha^{c} \geqslant E U_{H}(z)-\lambda p_{H}(z) \quad \forall z \in Z, \\
& \lambda \geqslant 0,
\end{aligned}
$$

where $\alpha^{c}$ and $\lambda$ are the shadow prices of the adding-up constraint (4.12) and the budget constraint (4.13).

Throughout the section we assume that optimal solutions for problems $\left(D_{c}\right)$ and $\left(P_{c}\right)$ exist and that the optimal values of these problems are identical. An analogous argument to the one used in Jerez (2003) implies that problems $\left(D_{c}\right)$ and $\left(P_{c}\right)$ have these properties provided (4.15) and (4.16) do not bind when $z$ is above a certain threshold $\bar{z} \in Z$. Intuitively, when consumption is sufficiently high, further increases in consumption must raise the price paid by the consumer than more than they raise the consumer's expected utility (for a given effort level). The competitive equilibrium price system derived at the end of this section has this property. The key assumption driving this result is that the marginal utility of consumption decreases asymptotically to zero.

By the complementary slackness theorem (see Krabs, 1979), a feasible solution $\left(x_{L}, x_{H}\right)$ for problem $\left(D_{c}\right)$ is optimal if and only if there exists $\left(\alpha^{c}, \lambda\right) \in \mathbf{R} \times \mathbf{R}_{+}$such that

$$
\begin{aligned}
& \lambda\left(\left\langle p_{L}, x_{L}\right\rangle+\left\langle p_{H}, x_{H}\right\rangle\right)=0, \\
& \alpha^{c} \geqslant E U_{L}(z)-\lambda p_{L}(z) \quad \forall z \in Z, \quad \text { with equality if } x_{L}(z)>0, \\
& \alpha^{c} \geqslant E U_{H}(z)-\lambda p_{H}(z) \quad \forall z \in Z, \text { with equality if } x_{H}(z)>0 .
\end{aligned}
$$

Condition (4.18) states that the optimal shadow price $\lambda$ is a complementary multiplier for the budget constraint (4.13). The monotonicity of preferences implies that $\lambda$ is positive, so the budget constraint holds with strict equality. ${ }^{17}$ Conditions (4.19) and (4.20) state that the optimal measures $x_{L}$ and $x_{H}$ are complementary multiplier vectors for the primal constraint systems (4.15) and (4.16). Here,

$$
E U_{i}(z)-\lambda p_{i}(z), \quad i=L, H,
$$

represents the expected consumer surplus from a deterministic contract which specifies effort $e_{i}$ and net trade $z$. Conditions (4.19) and (4.20) then imply that the optimal consumption plan $\left(x_{L}, x_{H}\right)$ puts all the probability weight on pairs of effort and net trade that maximize the expected consumer surplus. Moreover, the maximal expected consumer surplus is equal to $\alpha^{c}$ (the consumer's indirect utility). ${ }^{18}$

\footnotetext{
$\overline{17 \text { Suppose }} \lambda=0$. If $U_{i}$ is unbounded for some $i=L, H$, the corresponding primal constraint system is violated. If $U_{i}$ is bounded, the corresponding primal constraint system cannot hold with strict equality for any $z \in Z$ (since $U_{i}$ is strictly increasing). In either case, the support of $x_{i}$ is empty, so problem $\left(D_{c}\right)$ cannot have an optimal solution.

18 Problems $\left(D_{c}\right)$ and $\left(P_{c}\right)$ have the same optimal value.
} 


\subsection{Optimal production plans}

The firm chooses $y=\left(y_{L}, y_{H}\right) \in L$ to solve the following linear programming problem: $\left(D_{f}\right) \sup \left\langle p_{L}, y_{L}\right\rangle+\left\langle p_{H}, y_{H}\right\rangle$

s.t.

$$
\begin{aligned}
& -\left\langle E U_{L}, y_{L}\right\rangle+\left\langle E U_{H}, y_{L}\right\rangle \leqslant 0, \\
& \left\langle E U_{L}, y_{H}\right\rangle-\left\langle E U_{H}, y_{H}\right\rangle \leqslant 0, \\
& \left\langle r_{L}, y_{L}\right\rangle+\left\langle r_{H}, y_{H}\right\rangle \leqslant 0, \\
& y_{L}, y_{H} \geqslant 0 .
\end{aligned}
$$

The fact that $Y$ is a cone and $0 \in Y$ directly implies that an optimal production plan yields zero profits.

Lemma 4.1. Let $y$ be an optimal solution for problem $\left(D_{f}\right)$ then

$$
\langle p, y\rangle=\left\langle p_{L}, y_{L}\right\rangle+\left\langle p_{H}, y_{H}\right\rangle=0 .
$$

Let $q^{f}$ and $\left(\beta_{L}^{f}, \beta_{H}^{f}\right)$ denote the shadow prices of the technological constraint (4.23) and the incentive-compatibility constraints (4.21)-(4.22), respectively. By the complementary slackness theorem, a feasible solution $\left(y_{L}, y_{H}\right)$ for problem $\left(D_{f}\right)$ is optimal if and only if there exists $\left(q^{f}, \beta_{L}^{f}, \beta_{H}^{f}\right) \in \mathfrak{R}_{+}^{3}$ such that

$$
\begin{aligned}
& q^{f}\left(\left\langle r_{L}, y_{L}\right\rangle+\left\langle r_{H}, y_{H}\right\rangle\right)=0, \\
& \beta_{L}^{f}\left(\left\langle E U_{H}, y_{L}\right\rangle-\left\langle E U_{L}, y_{L}\right\rangle\right)=0, \\
& \beta_{H}^{f}\left(\left\langle E U_{L}, y_{H}\right\rangle-\left\langle E U_{H}, y_{H}\right\rangle\right)=0, \\
& 0 \geqslant p_{L}(z)-q^{f} r_{L}(z)-\beta_{L}^{f}\left(E U_{H}(z)-E U_{L}(z)\right) \quad \forall z \in Z, \\
& \quad \text { with equality if } y_{L}(z)>0, \\
& 0 \geqslant p_{H}(z)-q^{f} r_{H}(z)-\beta_{H}^{f}\left(E U_{L}(z)-E U_{H}(z)\right) \quad \forall z \in Z, \\
& \quad \text { with equality if } y_{H}(z)>0 .
\end{aligned}
$$

Conditions (4.25)-(4.27) state that the optimal shadow prices $q^{f}$ and $\left(\beta_{L}^{f}, \beta_{H}^{f}\right)$ are complementary multipliers for the technological constraint (4.13) and the incentivecompatibility constraints (4.21)-(4.22). Conditions (4.28) and (4.29) state that the optimal production plan $\left(y_{L}, y_{H}\right)$ puts all the weight on pairs of effort and net trade that maximize the producer surplus. Moreover, the maximal producer surplus is zero. The producer surplus from a deterministic contract which specifies effort $e_{i}$ and net trade $z$ is

$$
p_{i}(z)-q^{f} r_{i}(z)-\beta_{i}^{f}\left(E U_{j}(z)-E U_{i}(z)\right) \quad j \neq i, i, j=L, H .
$$

Take a high-effort contract. The price of the contract is $p_{H}(z)$, while its shadow cost is

$$
q^{f} r_{H}(z)+\beta_{H}^{f}\left(E U_{L}(z)-E U_{H}(z)\right) .
$$


The first term in (4.30) is a resource cost. That is, $r_{H}(z)$ is the average amount of the consumption good that the firm transfers to its customers under the contract, and $q r_{H}(z)$ is the shadow value of the transfer. The second term in (4.30) is an incentive cost (benefit). If the net trade $z$ is such that the customers prefer to shirk, the term reflects an incentive cost which is proportional to the utility gain from shirking. Conversely, if $z$ is such that the customers prefer not to shirk, the term reflects an incentive benefit which is proportional to the utility loss from shirking. If the customers are indifferent between conforming to the specification and shirking, the term is zero (so there is no incentive cost or benefit). A similar interpretation applies for low-effort contracts. ${ }^{19}$

\subsection{Competitive equilibrium prices and allocations}

The complementary slackness conditions for the firm's problem, (4.25)-(4.29), imply that the price of the contract offered by the firm is equal to the shadow cost of the contract. This result is analogous to the standard constant-returns condition that the price of a good is equal to its marginal cost of production. Let $x^{*}$ be the contract traded in a competitive equilibrium. Conditions (4.28) and (4.29) together with the market clearing condition imply that

$$
p_{i}^{*}(z)=q^{f *} r_{i}(z)+\beta_{i}^{f *}\left(E U_{j}(z)-E U_{i}(z)\right) \quad \text { if } x_{i}^{*}(z)>0, i=L, H,
$$

where $q^{f *}, \beta_{L}^{f *}$ and $\beta_{L}^{f *}$ are the optimal shadow prices of the firm's problem in a competitive equilibrium. Therefore, price of contract $x^{*}$ is

$$
\left\langle p^{*}, x^{*}\right\rangle=\left\langle p_{L}^{*}, x_{L}^{*}\right\rangle+\left\langle p_{H}^{*}, x_{H}^{*}\right\rangle,
$$

where

$$
\left\langle p_{i}^{*}, x_{i}^{*}\right\rangle=\left\langle q^{f *} r_{i}-\beta_{i}^{f *}\left(E U_{j}-E U_{i}\right), x_{i}^{*}\right\rangle .
$$

That is, the price of the contract traded in a competitive equilibrium is equal to its shadow cost. Lemma 4.1 and the market clearing condition moreover imply that

$$
\left\langle p^{*}, x^{*}\right\rangle=0 \text {. }
$$

That is, the price of the contract traded in a competitive equilibrium is equal to zero (i.e., the value of the expected net trade implied by the contract is zero).

Unlike the standard Arrow-Debreu model, the competitive equilibrium price system is not fully determined under moral hazard. This is a typical feature of models with a continuum of commodities, where the prices of commodities that are not traded in equilibrium are indeterminate. ${ }^{20}$ As a result there are many price systems that support a competitive equilibrium allocation. To see this, note that conditions (4.28) and (4.29) imply

$$
p_{i}^{*}(z) \leqslant q^{f *} r_{i}(z)+\beta_{i}^{f *}\left(E U_{j}(z)-E U_{i}(z)\right) \quad \forall z \in Z, i=L, H .
$$

\footnotetext{
19 Since the incentive-compatibility constraint with low effort does not bind at an incentive-efficient allocation, the first welfare theorem (see Theorem 4.1) implies that the shadow cost of a low-effort contract in a competitive equilibrium is a standard resource cost: $q^{f} r_{L}(z)$.

20 See Mas-Colell and Zame (1991).
} 
Therefore, the price of a contract $x$ that is not traded in a competitive equilibrium satisfies

$$
\left\langle p^{*}, x\right\rangle=\left\langle p_{L}^{*}, x_{L}\right\rangle+\left\langle p_{H}^{*}, x_{H}\right\rangle
$$

where

$$
\left\langle p_{i}^{*}, x_{i}\right\rangle \leqslant\left\langle q^{f *} r_{i}-\beta_{i}^{f *}\left(E U_{j}-E U_{i}\right), x_{i}\right\rangle .
$$

The price of a contract that is not traded in a competitive equilibrium is then lower that the shadow cost of the contract. If this was not the case, the firm could make infinite profits by supplying an infinite mass of one of these contracts.

A competitive equilibrium price system may be selected by taking the supremum over the set of prices $p \in P$ that satisfy conditions (4.31) and (4.35). The selected price system is

$$
p_{i}^{*}(z)=q^{f *} r_{i}(z)+\beta_{i}^{f *}\left(E U_{j}(z)-E U_{i}(z)\right) \quad \forall z \in Z, i=L, H .
$$

Under this price selection criterion, the price of a contract (whether traded or not) is equal to its shadow cost. This ensures that no small perturbation of an optimal production plan yields negative profits to the firm. ${ }^{21}$

In addition to the competitive equilibrium prices, we may characterize the competitive equilibrium allocations. The characterization is obtained by combining the complementary slackness conditions for the consumer's problem, (4.18)-(4.20), the complementary slackness conditions for the firm's problem, (4.25)-(4.29), and the market clearing condition. Proposition 4.1 summarizes the characterization of a competitive equilibrium.

Proposition 4.1. The allocation ( $\left.x^{*}, y^{*}\right)$ is a competitive equilibrium allocation if and only if $\left(x^{*}, y^{*}\right)$ is feasible, and there exist $\left(q^{f *}, \beta_{L}^{f *}, \beta_{H}^{f *}, \alpha^{c *}\right) \in \Re_{+}^{3} \times \Re$ and $\lambda^{*}>0$ such that

$$
\begin{aligned}
& q^{f *}\left(\left\langle r_{L}, x_{L}^{*}\right\rangle+\left\langle r_{H}, x_{H}^{*}\right\rangle\right)=0, \\
& \beta_{L}^{f *}\left(\left\langle E U_{H}, x_{L}^{*}\right\rangle-\left\langle E U_{L}, x_{L}^{*}\right\rangle\right)=0, \\
& \beta_{H}^{f *}\left(\left\langle E U_{L}, x_{H}^{*}\right\rangle-\left\langle E U_{H}, x_{H}^{*}\right\rangle\right)=0, \\
& \alpha^{c *} \geqslant E U_{L}(z)-\lambda^{*} q^{f *} r_{L}(z)-\lambda^{*} \beta_{L}^{f *}\left(E U_{H}(z)-E U_{L}(z)\right) \quad \forall z \in Z, \\
& \quad \text { with equality if } x_{L}^{*}(z)>0, \\
& \alpha^{c *} \geqslant E U_{H}(z)-\lambda^{*} q^{f *} r_{H}(z)-\lambda^{*} \beta_{H}^{f *}\left(E U_{L}(z)-E U_{H}(z)\right) \quad \forall z \in Z, \\
& \quad \text { with equality if } x_{H}^{*}(z)>0 .
\end{aligned}
$$

\footnotetext{
21 Alternatively, we could allow the firm to both sell and buy contracts. In this case, the complementary slackness conditions for the firm's problem directly pin down the price system in (4.38). Intuitively, (4.37) must hold with strict equality, for otherwise the firm could make infinite profits buying an infinite mass of contract $x$.

Under our price selection criterion, the equilibrium prices lie in a subset of the price space $P$ which is isomorphic to $\Re^{3}$. That is, an equilibrium price system is fully characterized by a triple $\left(\beta_{L}^{f *}, \beta_{H}^{f *}, q^{f *}\right) \in \mathfrak{R}_{+}^{3}$. The first welfare theorem (see Theorem 4.1) implies that $\beta_{L}^{f *}=0$ and $\beta_{H}^{f *}>0$ since only the incentive-compatibility constraint with high effort binds at an incentive-efficient allocation. Therefore, $p_{L}^{*}$ is linear and $p_{H}^{*}$ is non-linear.
} 
The competitive equilibrium price system $p^{*}=\left(p_{L}^{*}, p_{H}^{*}\right)$ (selected as the supremum over the set of equilibrium prices) is

$$
p_{i}^{*}(z)=q^{f *} r_{i}(z)+\beta_{i}^{f *}\left(E U_{j}(z)-E U_{i}(z)\right) \quad \forall z \in Z, i=L, H .
$$

Substituting the market clearing condition into the firm's complementary slackness conditions (4.25)-(4.27) gives (4.39)-(4.41). Substituting the market clearing condition and the firm's complementary slackness conditions (4.28)-(4.29) into the consumer's complementary slackness conditions (4.19)-(4.20) gives (4.42)-(4.43). The proposition states that conditions (4.39)-(4.43) are not only necessary but also sufficient for $x^{*}$ to be the contract traded in a competitive equilibrium. The complementary slackness condition (4.18) associated to the consumer's budget constraint is not included in the characterization. Since conditions (4.39)-(4.41) and (4.44) imply (4.18), this condition is redundant. The proof of the "if" statement is in Appendix A.

\subsection{Welfare theorems and existence}

The characterization of a competitive equilibrium allocation in Proposition 4.1 is equivalent to the characterization of an incentive-efficient allocation derived in Jerez (2003). Therefore, the two welfare theorems hold. ${ }^{22}$ Furthermore, the existence of optimal solutions to the planner's problem and its dual (established in Jerez, 2003) implies existence of a competitive equilibrium.

Theorem 4.1 (First Welfare Theorem). A competitive equilibrium allocation is incentive efficient.

Theorem 4.2 (Second Welfare Theorem). For any incentive-efficient allocation $(x, y)$ there is a price system $p \in P$ such that $(x, y, p)$ is a competitive equilibrium.

Theorem 4.3. A competitive equilibrium exists.

\section{Conclusion}

We have shown how to recover equilibrium prices supporting incentive-efficient allocations in a classic moral hazard economy. Our key modeling choice is to impose the incentive-compatibility constraints on firms, and not on consumers as in Prescott and Townsend (1984a). We have shown that equilibrium prices of insurance contracts are equal to the sum of the shadow costs arising from the resource and incentive-compatibility constraints in the planner's problem. The equilibrium allocations are the same as when the incentive-compatibility constraints are imposed on consumers. As in Prescott and Townsend (1984a), the two welfare theorems hold.

22 The First Welfare Theorem can also be derived using standard arguments. In Appendix A, we provide the proof for completeness. 
The results in this paper extend directly to the general class of economies with ex post asymmetric information analyzed by Prescott and Townsend (1984a, 1984b). This class includes economies with moral hazard and with ex post private information. To clarify the presentation, we have chosen one of their example economies to present our results. A key feature of the Prescott-Townsend class of economies is that the number of goods is finite. In particular, the economies are either static or have a finite time horizon. The number of unobservable actions (types) is also finite. This implies that, even though the dimension of the general space of lotteries where allocations lie is infinite, the number of resource and incentive-compatibility constraints is finite. In this paper, we exploit this semi-infinite structure and use the techniques of Linear Semi-Infinite Programming to derive our results.

We could have also considered a dynamic moral hazard economy with finitely many periods in the spirit of Rogerson (1985). ${ }^{23}$ There consumers experience an idiosyncratic endowment shock each period. Insurance contracts specify a sequence of time-indexed effort and net trade pairs, and must give consumers an incentive to conform to the effort specified each period. In period $t$ net trades are assigned contingent on the current state of the world, which is given by the sequence of realized idiosyncratic states from period 1 up to period $t$. Allowing for lotteries again renders the model linear. Dynamic incentivecompatibility constraints reflect the fact that the effort exerted in period $t$ affects the net trades assigned in all subsequent periods $t+1, \ldots, T$, because it affects the distribution of the shock at time $t$. Therefore, the effort exerted in period $t$ affects the consumers incentives to conform to the effort specifications in all subsequent periods $t+1, \ldots, T$. In a competitive equilibrium of the dynamic economy, prices reflect the fact that effort and net trade assigned in period $t$ imply a resource and an incentive cost in period $t$, as well as additional incentive costs in all subsequent periods $t+1, \ldots, T$.

Our results can also be extended to more general settings, including (infinite) dynamic economies. With an infinite time horizon, incentive-efficient allocations still solve a linear program (e.g. expected utilities and incentive-compatibility constraints are linear in the commodity bundles). The difference is that both the dimension of the space where allocations lie and the number of constraints in the planner's problem are now infinite. Extending our results thus requires (slightly more involved) Linear Infinite Programming techniques.

As noted by Prescott and Townsend (1984a), the extension of the Arrow-Debreu model to economies with adverse selection is not straightforward (see also Bisin and Gottardi, 2000, and Rustichini and Siconolfi, 2002). In a companion paper (Jerez, 2000), we suggest that the linear programming methodology can be helpful in understanding the problems which arise in decentralizing incentive-efficient allocations under adverse selection.

\section{Acknowledgments}

I am indebted to my advisor, Joe Ostroy, for his invaluable guidance. I am grateful to an editor and two referees for their comments and suggestions, as well as to Sushil Bikhchandani, Alberto Bisin, Berthold Herrendorf, Miguel Angel Goberna, Piero Got-

\footnotetext{
23 I thank a referee for suggesting dynamic extensions of the analysis.
} 
tardi, Birgit Grodal, David Levine, John Riley, Bill Zame, and participants of 8th World Congress of the Econometric Society (Seattle), SED Conference (Istanbul), European GE Conference (Paris), European Winter Meeting of the Econometric Society (London), Istituto Veneto's Economic Theory Workshop (Venice), SITE at Stanford, and seminars at UCLA, UBC, ITAM, Carlos III, UAB, Alicante, CERGE-EI, and CORE. Financial support from the Alfred P. Sloan Dissertation Fellowship, the Bank of Spain, and the DGCYT (projects SEJ2004-07861, BEC2002-00642, BEC2000-0172 and HI2001-0039) is gratefully acknowledged. Errors are mine.

\section{Appendix A}

\section{A.1. The linear programming problems}

The linear programs in Section 4 are known as Linear Semi-Infinite Programming (LSIP) problems. An LSIP problem is a linear program in which either number of variables or the number of constraints is finite (see Goberna and López, 1998). In this section, we set up the primal LSIP problem and derive its dual. The LSIP problems in Section 4 and Appendix A.2 obtain as particular cases of the problems in this section by applying the definitions in Table A.1.

\section{A.1.1. The primal problem}

Let $1 \leqslant m \leqslant n$ and $\Re^{n}$ be equipped with the Euclidean norm and partially ordered by means of the cone

$$
K_{m}^{n}=\left\{y \in \Re^{n}: y_{j} \geqslant 0, j=1, \ldots, m\right\} .
$$

Let $\omega \in \mathfrak{R}_{+}^{s}$ and define $Z=\left\{z \in \mathfrak{R}^{s}: z \geqslant-\omega\right\}$. Let $C(Z)$ denote the vector space of continuous real-valued functions on $Z$, endowed with the topology of uniform convergence on compact sets and partially ordered by means of the cone

$$
C_{+}(Z)=\{f \in C(Z): f(z) \geqslant 0 \forall z \in Z\} .
$$

The primal problem is to find $y \in \mathfrak{R}^{n}$ to solve

Table A.1

The primal and dual problems in Sections 4 and Appendix A.2

\begin{tabular}{llll}
\hline & The planner & The firm & The consumer \\
\hline$(n, m)$ & $(4,3)$ & $(3,3)$ & $(2,1)$ \\
$y$ & $\left(\beta_{L}, \beta_{H}, q, \alpha\right)$ & $\left(\beta_{L}^{f}, \beta_{H}^{f}, q^{f}\right)$ & $\left(\lambda, \alpha^{c}\right)$ \\
$c$ & $(0,0,0,1)$ & $(0,0,0)$ & $(0,1)$ \\
$b=\left(b_{L}, b_{H}\right)$ & $\left(E U_{L}, E U_{H}\right)$ & $\left(p_{L}, p_{H}\right)$ & $\left(E U_{L}, E U_{H}\right)$ \\
$f_{1}=\left(f_{1 L}, f_{1 H}\right)$ & $\left(-E U_{L}+E U_{H}, 0\right)$ & $\left(-E U_{L}+E U_{H}, 0\right)$ & $\left(p_{L}, p_{H}\right)$ \\
$f_{2}=\left(f_{2 L}, f_{2 H}\right)$ & $\left(0, E U_{L}-E U_{H}\right)$ & $\left(0, E U_{L}-E U_{H}\right)$ & $(\mathcal{I}, \mathcal{I})$ \\
$f_{3}=\left(f_{3 L}, f_{3 H}\right)$ & $\left(r_{L}, r_{H}\right)$ & $\left(r_{L}, r_{H}\right)$ & - \\
$f_{4}=\left(f_{4 L}, f_{4 H}\right)$ & $(\mathcal{I}, \mathcal{I})$ & - & - \\
\hline
\end{tabular}


(P) $\quad$ inf $\quad c \cdot y$

s.t. $A y \geqslant b, \quad y \in K_{m}^{n}$,

where $c \in \Re^{n}, b=\left(b_{L}, b_{H}\right) \in C(Z) \times C(Z)$, and $A: \Re^{n} \rightarrow C(Z) \times C(Z)$ is a continuous linear mapping. Problem $(P)$ is linear and has $n$ unknowns and infinitely many constraints.

\section{A.1.2. The dual problem}

Let $M_{c}(Z)$ denote the space of signed Borel measures on $Z$ which have compact support and are finite on compact sets. This space is the topological dual space of $C(Z)$.

Let $C(Z) \times C(Z)$ be paired in duality with $M_{c}(Z) \times M_{c}(Z)$. The reflexive space $\Re^{n}$ is paired with itself. The two pairings are endowed with their natural bilinear forms (to highlight the dimensionality of the spaces in the pairing we use the dot product and bracket notation for finite and infinite dimensions, respectively):

$$
\begin{array}{rlrl}
\langle f, x\rangle=\int_{Z} f_{L} \mathrm{~d} x_{L}+\int_{Z} f_{H} \mathrm{~d} x_{H}, & f & =\left(f_{L}, f_{H}\right) \in C(Z) \times C(Z), \\
& x=\left(x_{L}, x_{H}\right) \in M_{c}(Z) \times M_{c}(Z) ; \\
y \cdot z=\sum_{j=1}^{n} y_{j} z_{j}, & y \in \Re^{n}, z \in \Re^{n} .
\end{array}
$$

The adjoint of $A, A^{*}: M_{c}(Z) \times M_{c}(Z) \rightarrow \Re^{n}$, is defined by the relation

$$
y \cdot\left(A^{*} x\right)=\langle A y, x\rangle, \quad \text { for all } y \in K_{m}^{n}, x \in M_{c+}(Z) \times M_{c+}(Z) .
$$

We may write $A y=\sum_{j=1}^{n} y_{j} f_{j}$, where $f_{j}=\left(f_{j L}, f_{j H}\right) \in C(Z) \times C(Z)$ for $j=1, \ldots, n$. Then (A.1) can be expressed as

$$
y \cdot\left(A^{*} x\right)=\sum_{j=1}^{n} y_{j}\left\langle f_{j}, x\right\rangle, \quad \text { for all } y \in K_{m}^{n}, x \in M_{c+}(Z) \times M_{c+}(Z) .
$$

Write $A^{*} x \leqslant c$ as

$$
\sum_{j=1}^{n} y_{j}\left(\left\langle f_{j}, x\right\rangle-c_{j}\right) \leqslant 0, \quad \forall y \in K_{m}^{n}
$$

The dual problem is to find $x \in M_{c}(Z) \times M_{c}(Z)$ to solve

$$
\begin{array}{ll}
\text { (D) } \quad \text { sup } & \langle b, x\rangle \\
\text { s.t. } \quad & \left\langle f_{j}, x\right\rangle \leqslant c_{j}, \quad j=1, \ldots, m, \\
& \left\langle f_{j}, x\right\rangle=c_{j}, \quad j=m+1, \ldots, n, \\
& x \geqslant 0 .
\end{array}
$$

Problem $(D)$ is linear and has infinitely many unknowns and $n$ constraints. 


\section{A.2. Proof of Proposition A.1}

(a) The "only if" statement. Suppose $\left(x^{*}, y^{*}\right)$ is a competitive equilibrium allocation. Then $x^{*} \in X, y^{*} \in Y$ and $x^{*}=y^{*}$; i.e., $\left(x^{*}, y^{*}\right)$ is feasible. Let $\left(\alpha^{c *}, \lambda^{*}\right)$ and $\left(q^{f *}, \beta_{L}^{f *}, \beta_{H}^{f *}\right)$ denote the optimal shadow prices in the consumer's problem and the firm's problem, respectively. Then $q^{f *}, \beta_{L}^{f *}, \beta_{H}^{f *} \geqslant 0$ and $\lambda^{*}>0$. Substituting the market clearing condition into the firm's complementary slackness conditions (4.25)(4.27) gives (4.39)-(4.41). Substituting the market clearing condition and the firm's complementary slackness conditions (4.28)-(4.29) into the consumer's complementary slackness conditions (4.19)-(4.20) gives (4.42)-(4.43).

(b) The "if" statement. Suppose $\left(x^{*}, y^{*}\right)$ is feasible and there exist $\left(q^{f *}, \beta_{L}^{f *}, \beta_{H}^{f *}, \alpha^{c *}\right) \in$ $\Re_{+}^{3} \times \Re$ and $\lambda^{*}>0$ such that (4.39)-(4.43) hold. Feasibility implies $y^{*}=x^{*}$. It remains to show that $x^{*}$ solves both problem $\left(D_{c}\right)$ and problem $\left(D_{f}\right)$ for some choice of the price system. Let the price system be given by (4.44). Since $y^{*}=x^{*}$ and $y^{*} \in Y$, $x^{*}$ is a feasible solution for problem $\left(D_{f}\right)$. Conditions (4.39)-(4.41) and (4.44) moreover imply that $x^{*}$ and $\left(q^{f *}, \beta_{L}^{f *}, \beta_{H}^{f *}\right)$ satisfy (4.25)-(4.29). Hence, $x^{*}$ is an optimal solution for problem $\left(D_{f}\right)$. Since $x^{*} \in X$ and conditions (4.39)-(4.41) and (4.44) imply $\left\langle p^{*}, x^{*}\right\rangle=0$, it follows that $x^{*}$ is a feasible solution for problem $\left(D_{c}\right)$. Conditions (4.42) and (4.43) moreover imply that $x^{*}$ and $\left(\alpha^{c *}, \lambda^{*}\right)$ satisfy (4.19)-(4.20). Since $\left\langle p^{*}, x^{*}\right\rangle=0,(4.18)$ is also satisfied. Hence, $x^{*}$ is an optimal solution for problem $\left(D_{c}\right)$.

\section{A.3. Proof of Theorems 4.1, 4.2 and 4.3}

Incentive-efficient allocations are the Pareto-optimal allocations in the set of technologically feasible and incentive-compatible allocations. The problem of the planner is to find an insurance contract $\left(x_{L}, x_{H}\right) \in M_{c}(Z) \times M_{c}(Z)$ to solve

(D) $\sup \left\langle E U_{L}, x_{L}\right\rangle+\left\langle E U_{H}, x_{H}\right\rangle$

s.t.

$$
\begin{aligned}
& \left\langle\mathcal{I}, x_{L}\right\rangle+\left\langle\mathcal{I}, x_{H}\right\rangle=1, \\
& -\left\langle E U_{L}, x_{L}\right\rangle+\left\langle E U_{H}, x_{L}\right\rangle \leqslant 0, \\
& \left\langle E U_{L}, x_{H}\right\rangle-\left\langle E U_{H}, x_{H}\right\rangle \leqslant 0, \\
& \left\langle r_{L}, x_{L}\right\rangle+\left\langle r_{H}, x_{H}\right\rangle \leqslant 0, \\
& x_{L}, x_{H} \geqslant 0 .
\end{aligned}
$$

The primal problem is to find a quadruple $\left(\alpha, \beta_{L}, \beta_{H}, q\right) \in \mathbf{R}^{4}$ to solve

(P) $\inf \alpha$

s.t.

$$
\alpha \geqslant E U_{L}(z)-q r_{L}(z)-\beta_{L}\left[E U_{H}(z)-E U_{L}(z)\right] \quad \forall z \in Z,
$$




$$
\begin{aligned}
& \alpha \geqslant E U_{H}(z)-q r_{H}(z)-\beta_{H}\left[E U_{L}(z)-E U_{H}(z)\right] \quad \forall z \in Z, \\
& \beta_{L}, \beta_{H}, q \geqslant 0,
\end{aligned}
$$

where $\alpha$ is the shadow price of the adding-up constraint (A.3), $\beta_{L}$ and $\beta_{H}$ are the shadow prices of the incentive-compatibility constraints (A.4) and (A.5), and $q$ is the shadow price of the technological constraint (A.6).

Theorem A.1 below establishes the equivalence between the competitive equilibrium prices and allocations, on the one hand, and the solutions to problems $(D)$ and $(P)$, on the other hand.

\section{Theorem A.1.}

(i) Suppose $\left(x^{*}, y^{*}, p^{*}\right)$ is a competitive equilibrium. Let $\left(q^{f *}, \beta_{L}^{f *}, \beta_{H}^{f *}\right)$ and $\left(\alpha^{c *}, \lambda^{*}\right)$ be the optimal shadow prices in the problem of the firm and the consumer, respectively. Since $\lambda^{*}$ represents the marginal utility of money, the optimal shadow prices in the firm's problem can be measured in utils:

$$
\left(q^{*}, \beta_{L}^{*}, \beta_{H}^{*}\right)=\lambda^{*}\left(q^{f *}, \beta_{L}^{f *}, \beta_{H}^{f *}\right) .
$$

Then $x^{*}$ and $\left(\alpha^{c *}, q^{*}, \beta_{L}^{*}, \beta_{H}^{*},\right)$ are optimal solutions for problems $(D)$ and $(P)$, respectively.

(ii) Suppose $x$ and $\left(\alpha, q, \beta_{L}, \beta_{H}\right)$ are optimal solutions for problems $(D)$ and $(P)$, respectively. Let $y=x$ and define $p=\left(p_{L}, p_{H}\right)$ as

$$
p_{i}(z)=q r_{i}(z)+\beta_{i}\left(E U_{j}(z)-E U_{i}(z)\right) \quad \forall z \in Z, i=L, H .
$$

Then $(x, y, p)$ is a competitive equilibrium. Also, $\alpha$ represents the consumers' indirect utility in a competitive equilibrium.

Proof of Theorem A.1. An allocation $(x, y)$ is feasible if and only if $y=x$ and $x$ is feasible for problem $(P)$. Since $\lambda^{*}>0$, the conditions (4.39)-(4.43) that characterize a competitive equilibrium allocation in Proposition 4.1 can be restated as

$$
\begin{array}{ll}
q^{*}\left(\left\langle r_{L}, x_{L}^{*}\right\rangle+\left\langle r_{H}, x_{H}^{*}\right\rangle\right)=0, & \\
\beta_{L}^{*}\left(\left\langle E U_{H}, x_{L}^{*}\right\rangle-\left\langle E U_{L}, x_{L}^{*}\right\rangle\right)=0, & \\
\beta_{H}^{*}\left(\left\langle E U_{L}, x_{H}^{*}\right\rangle-\left\langle E U_{H}, x_{H}^{*}\right\rangle\right)=0, & \\
\alpha^{c *} \geqslant E U_{L}(z)-q^{*} r_{L}(z)-\beta_{L}^{*}\left(E U_{H}(z)-E U_{L}(z)\right) & \forall z \in Z, \\
\quad \text { with equality if } x_{L}^{*}(z)>0, & \\
\alpha^{c *} \geqslant E U_{H}(z)-q^{*} r_{H}(z)-\beta_{H}^{*}\left(E U_{L}(z)-E U_{H}(z)\right) \quad \forall z \in Z, & \\
\quad \text { with equality if } x_{H}^{*}(z)>0, &
\end{array}
$$

where $\left(q^{*}, \beta_{L}^{*}, \beta_{H}^{*}\right)=\lambda^{*}\left(q^{f *}, \beta_{L}^{f *}, \beta_{H}^{f *}\right) \in \mathfrak{R}_{+}^{3}$. Conditions (A.11)-(A.15) are the complementary slackness conditions for problems $(D)$ and $(P)$. This directly implies (i) and (ii). 
Parts (i) and (ii) in Theorem A.1 imply Theorems 4.1 and 4.2, respectively. Theorem 4.1 can also be derived using standard arguments.

Proof of Theorem 4.1. Suppose the contrary. That is, there is $\left(x^{\prime}, y^{\prime}\right) \in L^{2}$, with $x^{\prime} \in X$, $y^{\prime} \in Y, \quad x^{\prime}=y^{\prime}$, and $\left\langle E U, x^{\prime}\right\rangle>\left\langle E U, x^{*}\right\rangle$. The consumer's optimization condition (i) in Definition 4.1 then implies $\left\langle p^{*}, x^{\prime}\right\rangle>\left\langle p^{*}, x^{*}\right\rangle$. By feasibility, this is equivalent to $\left\langle p^{*}, y^{\prime}\right\rangle>\left\langle p^{*}, y^{*}\right\rangle=0$, which contradicts the firm's optimization condition (ii).

In Jerez (2003), we establish the existence of optimal solutions to problems $(P)$ and $(D)$. This result and Theorem A.1 imply Theorem 4.3.

\section{References}

Bennardo, A., Chiappori, P.A., 2003. Bertrand and Walras equilibria under moral hazard. Journal of Political Economy 11, 785-817.

Bisin, A., Gottardi, P., 1999. Competitive equilibria with asymmetric information. Journal of Economic Theory $87,1-48$.

Bisin, A., Gottardi, P., 2000. Decentralizing incentive efficient allocations of economies with adverse selection. Mimeo.

Bisin, A., Guatoli, D., 1997. Inefficiency of competitive equilibrium with asymmetric information and financial intermediaries. Discussion paper 1987. CEPR.

Goberna, M.A., López, M.A., 1998. Linear Semi-Infinite Optimization. Wiley, Chichester.

Gretsky, N.E., Ostroy, J.M., Zame, W.R., 1999. Perfect competition in the continuous assignment model. Journal of Economic Theory 88, 60-118.

Hewitt, E., 1959. Linear functionals on spaces of continuous functions. Fundamentae Mathematicae 37, 161-189.

B. Jerez, 2000. General equilibrium with asymmetric information: a dual approach. Working paper 510.02. UAB.

Jerez, B., 2003. A dual characterization of incentive efficiency. Journal of Economic Theory 112, 1-34.

Kehoe, T.J., Levine, D.K., Prescott, E., 2002. Lotteries, sunspots and incentive constraints. Journal of Economic Theory 107, 39-69.

Krabs, W., 1979. Optimization and Approximation. Wiley, New York.

Makowski, L., Ostroy, J.M., 1996. Perfect competition via linear programming. Mimeo.

Mas-Colell, A., Zame, W.R., 1991. Equilibrium theory in infinite dimensional spaces. In: Hildenbrand, W., Sonnenschein, H. (Eds.), Handbook of Mathematical Economics, vol. IV. Elsevier Science.

Prescott, E., Townsend, R., 1984a. Pareto optima and competitive equilibria with adverse selection and moral hazard. Econometrica 52, 21-45.

Prescott, E., Townsend, R., 1984b. General competitive analysis in an economy with private information. International Economic Review 25, 1-20.

Rogerson, W.P., 1985. Repeated moral hazard. Econometrica 53, 69-76.

Rothschild, M., Stiglitz, J.E., 1976. Equilibrium in competitive insurance markets: An essay on the economics of imperfect information. Quarterly Journal of Economics 90, 629-649.

Rustichini, A., Siconolfi, P., 2002. General equilibrium in economies with adverse selection. Mimeo.

Sun, Y., 1998. A theory of hyperfinite processes: the complete removal of individual uncertainty via exact LLN. Journal of Mathematical Economics 29, 419-503.

Wilson, C., 1977. A model of insurance markets with incomplete information. Journal of Economic Theory 16 , 176-207. 